\title{
Desafíos para pensar desde la vida en las ciencias sociales
}

\author{
María Luisa Eschenhagen ${ }^{1}$ \\ Universidad Pontificia Bolivariana, Medellín, Colombia. \\ Email: marialuisa.eschenhagen@upb.edu.co
}

\begin{abstract}
Resumen: Este texto pretende develar la ceguera de las ciencias sociales frente a las problemáticas ambientales del mundo, problemáticas que aparecen fehacientes e innegables en la actualidad. Esta ceguera se evidencia por ejemplo en la relativa ausencia del tema ambiental en el momento de proponer desafíos/retos de las ciencias sociales, como consecuencia de unas bases epistémicas que impiden pensar desde la vida y comprender la complejidad ambiental. Por lo tanto el artículo propone tres insumos, como lo son la colonialidad/modernidad, la complejidad y el pensamiento ambiental para pensar desde la vida, así como el sumak kawsay y el budismo para aproximarse a comprender la complejidad ambiental.

Palabras clave: ciencias sociales, modernidad/colonialidad, pensamiento ambiental, complejidad, sumak kawsay, budismo.
\end{abstract}

\section{Challenges to think springing from life in the social sciences}

\begin{abstract}
This paper aims to reveal the blindness of the social sciences towards dealing with the world's environmental problems, which appear irrefutable and undeniable today. This blindness is evident for example in the relative absence of environmental issues at the time of proposing darings/challenges from and to the social sciences, as a result of epistemic bases that prevent thinking from life and understand environmental complexity. Therefore the article proposes three inputs, which are coloniality/modernity, complexity and environmental thinking, to think out from life, as do the sumakkawsay and Buddhism to approach an understanding of environmental complexity.
\end{abstract}

Keywords: social sciences, modernity/coloniality, environmental thinking, complexity, sumakkawsay, Buddhism.

\section{Desafiospara pensar a partir davidanas ciências sociais}

Resumo: Este artigotem como objetivo revelara cegueira das ciênciassociaisperante as problemáticas ambientais do mundo, problemas que aparecemhojeirrefutáveis eincontestáveis.Essa cegueiraé evidente, por exemplo, na ausênciarelativa dequestões ambientaisno momentode propordesafiosdas ciências sociais, como resultado debasesepistemológicasque impedem apensar a partir davidae entendera complexidade ambiental. Portanto, oartigo propõetrês entradas, como são a colonialidade/modernidade, a complexidade eo pensamento ambientalpara pensar a vida, assim como o sumakkawsayeo budismopara nos aproximar nacompreensão da complexidadeambiental.

Palavras-chave: ciências sociais,modernidade/colonialidade, pensamento 
ambiental, complexidade, sumakkawsay, Budismo.

Actualmente gran parte del mundo se caracteriza por estar cada vez más sobredeterminado por decisiones administrativasorientadas a cumplir con metas utilitaristas y de acumulación de capital, a través de una racionalidad instrumental. En términos de Deneault (2013), se trata de un mundo dominado por la "revolución anestesiante", la gestión totalitaria, o también denominada gobernanza.Los espacios, ya sea en las aulas, en las comunidades académicas, en los espacios públicos e independientemente de las áreas de conocimiento,para pensar, disentir y debatir son cada vez más escasos. En este contexto los conocimientos provenientes de las ingenierías y ciencias duras, así como de las administrativas y económicas, y más específicamente financieras,son los que intervienen y transforman de manera significativa y profunda todos los aspectos de la vida, es decir en su conjunto las condiciones socioambientales.Se trata de un mundo gobernado ya no por ideas políticas, sino por principios económicos dominados por el capitalismo financiero. Como bien dice Balandier (2015:119), es preciso reconocer la "supremacía de la economía liberal, con sus instituciones híbridas con una forme de democracia parlamentaria asociada a aquella de un poder tecnocrático”.

Hace mucho tiempo que la filosofía ha sido marginadaen las universidades y las ciencias sociales son sistemáticamente subestimadas, subfinanciadas, y utilizadas de manera instrumental para implementar y justificar decisiones en las que priman factores económicos,decisiones desarrollistas, neoliberales. Y por lo general,en las universidades y los espacios públicos y de decisión olvidan, ignoran o desconocen los múltiples roles que tienen los profesionales de las ciencias socialesen la sociedad, entre los cuales se cuentan, como bien lo señala Serje (2013),

[el de] "críticos", para poner en evidencia la dimensión social y política de las intervenciones; el de "expertos" para contrarrestar el nosaber y sus implicaciones o "consultores" para iluminar creativamente las dimensiones que los técnicos y planificadores insisten en ignorar; el de "comunicadores" para movilizar conciencias y "activistas" para aportar herramientas que hagan visibles otras formas de vida social. Y, ante todo, el de testigos, para dar cuenta de las secuelas que, como la hojarasca, va dejando a su paso el avance de las locomotoras.

Es decir, el científico social tiene un gran potencial para aportar a la construcción organizativa, productiva, cultural y por supuesto ambiental, de la sociedad.Sin embargo, no ha logrado obtener el lugar ni el reconocimiento merecido. Por lo anterior, y considerando la interdependencia entre teoría y práctica, surge la inquietud acerca de si las preguntas y los marcos epistémicos, teóricos, que predominan en las ciencias sociales, en parte no 
han propiciado esta misma situación, lo cual lleva a cuestionar:¿las reflexiones teóricas de las ciencias sociales fortalecen, remiendan o critican el sistema hegemónico?De ahí la pertinencia delas dos preguntas convocantes de este número especial de la Revista POLIS: ¿Cuáles son los principales desafíos que enfrentarán las Ciencias Sociales en nuestro continente? ¿Cuáles son las principales preguntas que deberemos hacernos para contribuir al futuro de la democracia, de la paz y de la sustentabilidad en nuestras sociedades?

Por mi trayectoria académica, enmarcada en el estudio de la problemática ambiental desde las ciencias sociales, las presentes reflexiones se enfocarán primero, brevemente, sobre cómo se percibe el aspecto ambiental en los desafíos y retosenunciados de las ciencias sociales, para luego revisar cuáles podrían ser las preguntas para pensar la sustentabilidad, desde la vida, en las ciencias sociales y desde ellas.

Para iniciar el ejercicio analicé veintitrés textos que se dedican a reflexionar explícitamente sobre los retos y desafíos de las ciencias sociales ${ }^{2}$. Un primer aspecto que llama la atención es la debilidad de interpretación del contexto socioambiental y conceptual que se encuentra en los textos considerados, cuando abordan el análisis de los problemas ambientales imperantes, innegables en todas las escalas (regional, nacional, internacional $^{3}$ ) y muchas veces con derivaciones en términos de conflictividad social (en aumento casi exponencial) ${ }^{4}$.

Esta debilidad salta a la vista, por ejemplo, ante la ausencia deltérmino ambiente (y/o medio ambiente):que en doce textos ni se menciona, en solo seis se alude, sin profundizar,a la existencia de problemas ambientales,y en cuatrose lo utiliza únicamente como un adjetivo más, entre otros. Solo uno de los textos, el de Walsh (2007), presenta una mayor conceptualización al respecto. Sucede igual cuando se revisa el uso de los conceptos natural o naturaleza: en cuatro textos no aparecen, en siete simplemente resultan ser un adjetivo, en uno se refiere arecursos naturales y en seisa las ciencias naturales, y en cuatro se le asimila como ecosistema.Solo un texto,el de De Sousa Santos (2007), realiza una conceptualización al respecto, y Walsh también se aproxima a proponer una colonialidad de la naturaleza. Llama además la atención que en tres de los textos se retoma la idea ancestral occidental de la dominación sobre la naturaleza.

Este panorama resulta muy revelador, muestra con certeza y claridad las dificultades que tienen las ciencias sociales para pensar los problemas socioambientales. En el mejor de los casos (siete de veintitrés) se reconoce que existe un problema, riesgo, vulnerabilidad o degradación ambiental, pero sin que dicho problema conlleve a consecuencias coherentes sobre el quehacerde las ciencias sociales. El ambiente y la naturaleza por lo tanto siguen concibiéndose como aspectos que están "ahí afuera”, comoseparados de la sociedad. Solo dos textos se ocupan del tema ambiental y ninguno lo relaciona directamente como un desafío de las ciencias sociales. Este panorama resulta preocupante. 
Sin embargo, si se revisa la relación histórica entre las ciencias sociales y la comprensión del entorno natural, así como los fundamentos epistémicos para identificar y pensar la vida, éstas ausencias y dificultades aparecen más como una consecuencia lógica que como unacasualidad (ver también Eschenhagen, 2012). Hasta se podría hablar de una ceguera ambiental de las ciencias sociales. Como bien lo señala Leff $(2014,19)$ "la crisis ambiental abre una crítica sobre una falla fundamental de las ciencias sociales: el haber pensado el orden social independientemente de las condiciones de la naturaleza en las que se constituye, funciona y se autodestruye la sociedad”. Esta ceguera ambiental tiene causas y raíces epistemológicas. Las ciencias sociales nacieron dentro de la modernidad, ya marcadaspor un claro dualismo y una escisión entre cultura y naturaleza, cuerpo y mente, sujeto-objeto, así como dentro de la racionalidad mercantil naciente.

Es decir, existen unas formas muy específicas de posibilitar, construir y validar el conocimiento en las ciencias sociales que impiden reconocer, pensar y considerar las condiciones de la vida, la naturaleza. Es preciso aquí donde están los retos y desafíos más grandes de las ciencias sociales: ¿Cómo reconfigurar las bases epistémicas de las ciencias sociales para comprender y pensar la complejidad socioambiental?Responder implica también cuestionar el conocer para qué y para quién,y a su vez plantea la pregunta ¿qué es importante pensar? Responder estos interrogantes implicaabrir espacios amplios de discusión, ya que sus respuestas, que no serán únicas sino diversas y múltiples, solo se podrán dar en espacios de debate abierto, donde se avanza a través de la controversia, la discusión, la complementariedad. Pero estos espacios de pensar, espacios de naturaleza más bien filosófica, han sido reducidos, eliminados o marginados en el mundo moderno,el cual está más interesado por responder a las preguntas del cómo funcionan las cosas, en vez del por qué y para qué de los fenómenos.

A la vez, no se pueden desconocer los importantes avances en las propias ciencias sociales,en sus nuevos campos, por ejemplo:educación ambiental, historia ambiental, antropología ambiental,sociología ambiental,ecología política, economía ecológica, etc., avances que se hacen visiblesa través de asociaciones regionales latinoamericanas ${ }^{5}$, de la presencia ambiental en eventos centrales de las ciencias sociales como la CLACSO, en la formación de posgrados (ver Eschenhagen 2009), etc. Algunos de estos nuevos campos ya tienen una trayectoria de décadas. Sin embargo, regresa la pregunta, al observar, por un lado, que el desarrollo de estos campos no ha logrado permear suficientemente las ciencias sociales para ser considerados como un desafío para estas y, por el otro, que, a pesar de políticas ambientales implementadas desde 1972 y más concretamente desde el Informe Brundtland de 1987 y la Agenda 21 de 1992 a nivel mundial, no hay mejoras significativas en el ámbito ambiental, sino que se asiste más bien a una deterioración constante, sistemática y masiva. Entonces resulta indispensable preguntarse por las causas más profundas de las problemáticas socioambientales. 
Es aquí donde entran los retos epistémicos para leer los fenómenos socioambientales complejos y comenzar a preguntarse con qué lógicas, racionalidades y conocimientos se construyeron y justificaron estas políticas que al parecer no logran ser sustentables a largo plazo. De manera general se puede responder que como sociedades occidentales somos hijos de la modernidad, con la cual el territorio ha sido transformado significativa y radicalmente. Leff señala claramente que

la crisis ambiental es el signo y el síntoma más fuerte de ese límite de la modernidad. La crisis ambiental es en el fondo la manifestación de los modos dominantes y hegemónicos de organización del mundo moderno. Es en este sentido radical que la cuestión ambiental llama a una reconstrucción de las ciencias sociales y una refundamentación de los modos de habitar el mundo. $(2014,27)$.

Ahora, para abrir y reconstruir las ciencias sociales, volviéndolas capaces de comprender y pensar la complejidad ambiental 6 , considero importante forjartresinsumos necesarios. Antes de presentarlos, me interesa señalar que no se trata de insumos que puedan funcionar por separado, o combinados en forma indiscriminada, ya que tomados así, pueden resultar epistemológicamente incompatibles.Se trata de aproximaciones que ofrecen reflexiones teóricas críticas e inquietudes que invitan a pensar los problemas socioambientales desde otras perspectivas a las tradicionalmente planteadas por las ciencias sociales, a partir de las cuales se pueden dar lecturas y soluciones alternativas ${ }^{7}$.

El primer insumo propone la consideración de los aportes que puede ofrecer toda la vertiente desarrollada por pensadores latinoamericanos en torno a la modernidad/colonialidad, a través de sus conceptos centrales, la colonialidad del poder, la colonialidaddel saber y la colonialidaddel ser. Son representantes de esta vertiente Aníbal Quijano, Catherine Walsh, Santiago Castro-Gómez, Edgardo Lander, Walter Quijano, Ramón Grosfoguel, Eduardo Restrepo, Olver Quijano y muchos más. Sus reflexiones y aportes han llevado también a problematizar la geopolítica del conocimiento en la que reina una hegemonía epistémica (ver, Mignolo, 2002). Esto resulta importante para reflexionar sobre las potencialidades y limitaciones de los conceptos y teorías utilizadas y sus fundamentaciones eurocéntricas, así como para visibilizar y evidenciar los lugares de enunciación y sus implicaciones. Así, la perspectiva de la modernidad/colonialidad sirve, como bien dice Walsh $(2007,104)$

por un lado, como perspectiva para analizar y comprender los procesos, las formaciones y el ordenamiento hegemónicos del proyecto universal del sistema-mundo [... ] y, por el otro, para visibilizar, desde la diferencia colonial, las historias, subjetividades, conocimientos y lógicas de pensamiento y vida que desafían esta hegemonía.

El segundo insumo concierne a la necesidad de pensar desde la vida. Para aproximarse a este reto considero valioso los aportes de dos autores, 
distintos entre sí. Mientras que uno, Enrique Leff,realiza una búsqueda desde una perspectiva filosófica y a lo largo de su obra va construyendo nuevos conceptos para leer los fenómenossocioambientales, el otro, Carlos Maldonado, busca el potencial de las ciencias de la complejidad para pensar desde la vida.

No es lo mismo pensar desde un objeto, inerte, previsible, característico de la modernidad, a pensar desde la vida la cual resulta ser dinámica, impredecible, diversa como lo están planteando las ciencias de la complejidad (Maldonado, 2010). En este sentido Maldonado (2013) primero pone en claro que la propia denominación de ciencias sociales es decimonónica y no necesariamente conserva pertinencia para los tiempos actuales. Las divisiones disciplinarias resultan ya ser insosteniblesfrente a los problemas complejos de la sociedad que consisten en el entrelazamiento del sistema natural, humano y artificial. De ahí que los sistemas sociales ya no se pueden explicar en términos de equilibrio, fuerza, control, movimiento, estabilidad, causalidad, etc. En lugar de ellos,habrá que pensar en términos cambiantes, fluctuantes, estocásticos,autoorganizativos, sinérgicos. Estos sistemas complejos se abordan,por ejemplo, desdela evolución y adaptación, el trabajo en red, el comportamiento colectivo, las dinámicas no-lineales o la termodinámica del no equilibrio, lo cual ya se puede observar en el Instituto Santa Fe (http://www.santafe.edu/ ) que trabaja en torno a la fundamentación teórica de las ciencias de la complejidad y el New England Complex Systems Institute (NECSI) (http://necsi.edu/) quienes se han enfocado más en la solución de problemas. El aporte valioso de las ciencias de la complejidad, que son ciencias de lo posible, es que claramente rompen con los tres atributos de la humanidad occidental que son, de acuerdo con Maldonado, la legalidad, el determinismo y la reversibilidad, reconociendo más bien los atributos de la naturaleza, como lo son lo caótico y lo irregular, y ofrecen entendimientos completamente nuevos sobre la vida ${ }^{8}$.

Desde una perspectiva más filosófica, Enrique Leff plantea que las formas de conocer de la civilización moderna occidental, basadas sobre la ciencia moderna, incapaz de comprender la complejidad ambiental, y con las cuales ha transformado el mundo, han resultado ser insustentables. Se trata de un conocimiento que ha posibilitado una "racionalización de la vida que ha generado la hiperrealidad de un mundo sobre-economizado, sobreobjetivado y cosificado [y por ende] una política desarraigada de la tierra” (Leff, 2010:15). Frente a este problema, Leff viene elaborando y concretando a lo largo de más de 20 años, y de un sinfín de artículos y libros, conceptos tan centrales como saber ambiental, racionalidad ambiental y epistemología ambiental, que en su conjunto conllevan, a través del diálogo de saberes, a la complejidad ambiental. En 2002 Leff fue el impulsor intelectual para escribir y promover el Manifiesto por la vida, una ética para la sustentabilidad, que se presentó como posición latinoamericana en la Cumbre ambiental mundial de Johannesburgo en el 2002. Allí plasmó y sintetizó aspectos centrales del saber y la racionalidad ambiental. En su último libro, La apuesta por la vida (2014), después de realizar una crítica dura y muy fundamente a la sociología clásica, Leff propone una sociología ambiental 
para posibilitar una sustentabilidad de la vida. Tal vez en la siguiente cita puede verse mejor lo que significa pensar desde un pensamiento ambiental:

soy un ser en el mundo, conviviendo con otros, un nosotros inscrito en la trama de la vida, atrapado por las circunstancias de mi existencia, arrastrado por la muerte entrópica del planeta; o un ser-siendo en la heterogénesis de un mundo en vías de diversificación, construyendo un mundo, desde los principios de la vida, para la sustentabilidad de la vida (Leff, 2010, 27).

Se trata de reflexiones que el mundo moderno tendrá que recuperar, redescubrir, pero que en otras cosmovisiones resultan estar presentes, con lo cual paso al tercer insumo.Este último insumo apunta a inspirarse y dejarse permear por los aportes y las reflexiones que pueden ofrecer el sumakkawsay o suma qamaña de los Andeso la economía budista de Asia. Se trata decosmovisiones no modernas que claramente no establecen la escisión tajante de cultura-naturaleza, como lo realiza el mundo moderno. Se trata de dos cosmovisiones de lugares muy distantes pero que tienen elementos parecidos. Así, los aymara dicen que "saber vivir implica estar en armonía con uno mismo; “estar bien” o “sumanqaña” y luego, saber relacionarse o convivir con todas las formas de existencia... “suma qamaña”... significa vivir en armonía y equilibrio; en armonía con los ciclos de la Madre Tierra, del cosmos, de la vida y de la historia, y en equilibrio con toda forma de existencia.” (HuanacuniMamani, 2010, 19), y

BuddhadasaBhikku put it well: The entire cosmos is a cooperative. The sun, the moon, and the stars live together as a cooperative. The same is true for humans and animals, trees and soil. Our bodily parts function as a cooperative. When we realize that the world is a mutual, interdependent, cooperative enterprise, that human beings are all mutual friends in the process of birth, old age, suffering, and death, then we can build a noble, even heavenly environment. If our lives are not based in this truth, then we shall all perish.Sivaraksa $(2002,13)$.

Es a partir de estas concepciones que construyen sus epistemologías y formas específicas de ser y estar en el mundo.Como bien lo señala Descola (2012), ellasllevan a otras formas de habitar. Y es de señalar que no se trata de idealizar al "buen salvaje”, ni de buscar conversiones, que difícilmente serán posiblesde emprender por los“hijos de la modernidad”. Más bien se trata de conocer, pensar, discutir y dejarse inspirar, desde otros marcos filosóficos y epistémicos, para repensar y reconfigurar otros mundos posibles, desde las propias posibilidades y condiciones.

Se trata de insumos, que en su conjunto inspiran, incentivan y ayudan a romper la visión de mundo moderna, capitalista, hegemónica, la cual ampliamente ha demostrado ser insustentable para la vida. Es indispensable cambiar esa visión de mundo.

Por lo tanto, si el eje central de preocupación es efectivamente la 
reproducción y sustentabilidad de la vida, el desafío central es pensar la vida desde las ciencias sociales. Si el siglo XX estuvo marcado por la preocupación y el pensamiento en torno al progreso y el desarrollo, el siglo XXI debe estar marcado por pensar desde y para la vida. Para ello se requiere de creatividad, capacidad de asombro y pensamiento crítico y reflexivo. 


\section{Notas}

1 Profesora investigadora de la Escuela de Ciencias Sociales de la Universidad Pontificia Bolivariana, sede Medellín, Colombia, coordinadora del Grupo Territorio. www.pensamientoambiental.de

${ }^{2}$ Los textos se buscaron con las palabras claves de "ciencias sociales" + “desafío” y “ciencias sociales” + “reto”. Se eligieron los textos de revistas académicas, así como tres entrevistas (ver anexo 1).

${ }^{3}$ Están p.ej. el informe de Stern, del año 2006, sobre las implicaciones del cambio climático, quien advierte que si no se invierte ahora el 1\% del PIB mundial en proyectos para mitigar los efectos del cambio climático, las consecuencias negativas de éste podrán costar en un futuro cercano hasta un $20 \%$ del PIB mundial. El informe de la World Wildlife Foundation (WWF), del 15 de mayo del 2012, titulado "The Living Planet Report 2014”, documenta el estado altamente crítico de la huella ecológica y sus implicaciones p.ej. sobre la pérdida de biodiversidad y la sobrepesca de los mares. También están los informes nacionales sobre la pérdida de la biodiversidad, como p.ej. en Colombia, de entidades gubernamentales u otros (en Colombia p.ej. la Contraloría (2012) o por parte de independientes (Rodríguez Becerra (2012)).

4 Observatorio de conflictos mineros en América Latina http:// www.conflictosmineros.net/ (consultado 10.08.2015), Observatorio de conflictos socioambientales (Argentina) http://www.unrc.edu.ar/unrc/uniysoc/obspsocamb.php (consultado 10.08.2015), Observatorio Latinoamericano de conflictos ambientales http://www.olca.cl/oca/index.htm (consultado 10.08.2015)

5 Ver p.ej.: Associação Nacional de Pós-Graduação e Pesquisa em Ambiente e Sociedade (ANPAS), Centro Latinoamericano de Desarrollo Sustentable (CLADES), Foro Latinoamericano de Ciencias Ambientales (FLACAM), Grupo de Trabajo de Ecología Política (CLACSO), Red Iberoamericana de Economía Ecológica (REDIBEC), Rede de Pensamiento Ambiental, Sociedad Científica Latinoamericana de Agroecología (SOCLA), Sociedad Latinoamericana y Caribeña de Historia Ambiental (SOLCHA).

${ }^{6}$ Considerando que esta complejidad ambiental: "no remite a un todo -ni a una teoría de sistemas, ni a un pensamiento holístico multidimensional, ni a la conjunción y convergencia de miradas multirreferenciadas. Es, por el contrario, el desdoblamiento de la relación del conocimiento con lo real que nunca alcanza totalidad alguna, lo que disloca, desborda y desplaza la reflexión epistemológica desde el estructuralismo crítico hasta el reposicionamiento del ser en el mundo en su relación con el saber. [...] La complejidad ambiental emerge de la hibridación de diversos órdenes materiales y simbólicos que, determinada por la racionalidad científica y económica, ha generado este mundo objetivado y cosificado que se va haciendo resistente a todo conocimiento." (Leff, 2007, 50)

7 El término alternativa, es utilizado con mucha frecuencia, por lo cual valdría la pena especificarlo, ver para ello Rojas-mora (2009).

8 Ver proyectos de investigaciones como p.ej. sobre la evolución de la complejidad y la inteligencia en la tierra: http://www.santafe.edu/templeton/evolution-complexity/o sobre Ciudades, escalas y sostenibilidad http://www.santafe.edu/research/cities-scaling- 
Polis, Revista Latinoamericana, Volumen 14, $N^{\circ} 41,2015$

and-sustainability/ que se aproximan de una manera muy particular, desde la biología, evolución, neurología, física, química y también las ciencias sociales para comprender estos problemas. En estas investigaciones claramente se disuelve la separación entre lo físico y lo vivo, entre lo natural y lo cultural. 


\section{Bibliografía}

Balandier, Georges (2015), Recherche du politique perdu, Ed. Fayard, Francia.

Deneault, Alain (2013), Gouvernance. Le management totalitaire, Ed. Lux, Montreal.

Descola, Philippe (2012 [2005]), Más allá de naturaleza y cultura, Ed. Amorrortu, Buenos Aires.

Eschenhagen, María Luisa (2009), Educación ambiental superior en América Latina, Retos epistemológicos y curriculares, Red Colombiana de Formación Ambiental, Universidad de Ciencias Aplicadas y Ambientales, ECOE Ediciones.

Ídem (2012), “Contexto y exigencias a las ciencias sociales para afrontar los problemas ambientales”, En: Revista Polis, Chile, $N^{\circ}$ 30, enero, http:// polis.revues.org/2341(consultado 4.8.15).

Huanancuni Mamani, Fernando (2010), Buen vivir / vivir bien, filosofía, políticas, estrategias y experiencias regionales andinas, http://es.scribd.com/ doc/64246135/Buen-Vivir-Fernando-Hunacuni-Mamani\#scribd (consultado 4.8.15).

Leff, Enrique (2007), “La complejidad ambiental”, En: Gaia Scientia 2007, 1(1): 47-52.

Ídem (2010), "El desvanecimiento del sujeto y la reinvención de las identidades colectivas en la era de la complejidad ambiental”. En: Polis Revista Latinoamericana, Nr. 27 http://polis.revues.org/862 (consultado 4.8.15)

Ídem (2014), La apuesta por la vida, Siglo XXI, México.

Maldonado, Carlos (2010), “Sistemas vivos y complejidad”, en: Eschenhagen, María Luisa (comp.) (2010), Aportes ambientales desde América Latina para la apertura de las ciencias sociales, libro compilado, Universidad Central.

Manifiesto por la vida, por una ética para la sustentabilidad (2002), http:/ /www.scielo.br/pdf/asoc/n10/16893.pdf (consultado 4.8.15).

Mignolo, Walter (2002), “Colonialidad global, capitalismo y hegemonía epistémica”, En: Walsh, Catherine (2002), Indisciplinar las ciencias sociales, geopolíticas del conocimiento y colonialidad del poder. Perspectivas desde lo andino, Ed. AbyaYala, Quito.

Observatorio de conflictos mineros en América Latina http:// www.conflictosmineros.net/ (consultado 10.08.2015). 
Polis, Revista Latinoamericana, Volumen 14, $N^{\circ}$ 41, 2015

Observatorio de conflictos socioambientales (Argentina) http:// www.unrc.edu.ar/unrc/uniysoc/obspsocamb.php (consultado 10.08.2015).

Observatorio Latinoamericano de conflictos ambientales http://www.olca.cl/ oca/index.htm (consultado 10.08.2015).

"Pertinencia y calidad: la Facultad de Ciencias sociales frente a los retos de Colombia” (2013), Revista Universidad de los Andes, SERJE, Colombia.

Rojas-mora, Sneider (2009), “Aproximaciones al concepto de alternativas, provocaciones para pensar desde lo simple, lo complejo”, en: Eschenhagen, María Luisa y Carlos Eduardo Maldonado (editores académicos), Un viaje por las alternativas al desarrollo, perspectivas y propuestas teóricas, Ed. Universidad Pontificia Bolivariana y Universidad del Rosario.

Serje, Margarita (2013), Ciencias sociales y locomotoras, en Latitudeshttp:/ /sextante.uniandes.edu.co/index.php/ejemplares/sextante-2/latitudes/ciencias-sociales-y-locomotoras (consultado 4.8.15).

Sivaraksa, Sulak (2002), "Economic Aspects of Social and Environmental Violence from a Buddhist Perspective Author(s)”. En: Buddhist-Christian Studies, Vol. 22 University of Hawai'i Press Stable URL: http:// www.jstor.org/stable/1390560 (consultado 4.8.15).

\section{Anexo 1}

Aguilera Hintelholher, Rina Marissa (2014, enero-abril), “Complejidades impredecibles: desafíos de las Ciencias Sociales en el mundo contemporáneo”. Estudios Políticos N³1, 129-146. México, D.F. Recuperado de http:/ /www.revistas.unam.mx/index.php/rep/article/view/44116(consultado 10.08.2015).

Bas Amorós, Josep Enric (2002), Nuevos retos, viejos enfoques, necesidad repensar las Ciencias sociales, aportes desde la sociología. Actas del III Simposio de Historia Actual.Vol 1, Recuperado de http://dialnet.unirioja.es/ servlet/articulo?codigo=793161(consultado 10.08.2015).

Castillo F., Dídimo (2004, enero-junio), “La Sociedad global y los desafíos para las ciencias sociales”. Revista Venezolana de Ciencias Sociales. Vol. 8, $\mathrm{N}^{\circ}$ 1. Universidad Nacional Experimental Rafael María Baralt, Venezuela. Recuperado de http://www.redalyc.org/articulo.oa?id=30980102(consultado 10.08.2015).

De Sousa Santos, Boaventura (29 de marzo de 2007), Los desafíos de las ciencias sociales hoy. CLACSO, Argentina. 101-120. Recuperado de http:/ /biblioteca.clacso.edu.ar/ar/libros/coedicion/boavent / cap\%203.pdf(consultado 10.08.2015).

González Casanova, Pablo (1991, diciembre), “Los desafíos de las ciencias 
sociales, hoy”. Revista Universidad de México N (491), 23-25. Recuperado de http://www.revistadelauniversidad.unam.mx/ojs_rum/index.php/rum/ article/view/13491/14729 (consultado 10.08.2015).

Hernández Cifuentes, Yhoban Camilo (2013-10-28), “Ayudar a consolidar procesos democráticos: reto de las ciencias sociales en América Latina”. Rebelión. Recuperado de http://www.rebelion.org/noticia.php?id= 176026(consultado 10.08.2015).

Herrera, José Darío (2010), “La investigación en las ciencias sociales: breve historia y retos actuales”. Revista de la Universidad de la Salle N ${ }^{\circ}$ (51), 5570. Recuperado de http://es.scribd.com/doc/145563398/Jose-Dario-HerreraLa-investigacion-en-las-ciencias-sociales-Breve-historia-y-retosactuales\#scribd(consultado 10.08.2015).

Luján, Hernando (18 de junio de 2012), Las Humanidades y las Ciencias Sociales son necesarias en el México de hoy. Entrevista con Rosalba Casas y Guillermo Hurtado. Programa de radio Perfiles, emitido por de Radio UNAM. México. Recuperado de http://www.revista.unam.mx/vol.14/num1/ art06/(consultado 10.08.2015)Maldonado Carlos Eduardo (20013) Significado e impacto social de las ciencias de la complejidad http:// www.carlosmaldonado.org/articulos/Significado\%20e\%20impacto\% 20social\%20de\%20las\%20ciencias\%20de\%20la\%20complejidad.pdf (consultado 10.08.2015).

Mercado Maldonado, Asael (2011), “Ciencias sociales: retos y tendencias temáticas sociales”. Revista deCiencias Sociales. $N^{\circ} 133-134$, 57-73. Costa Rica: Universidad de Costa Rica. Recuperado http://www.redalyc.org/pdf/ 153/15323589005.pdf(consultado 10.08.2015).

Ministerio de Educación de Colombia (S.f), La formación en ciencias: ¡el desafío! Estándares básicos de competencias en ciencias sociales y ciencias naturales. 97-147. Recuperado de http://www.mineducacion.gov.co/1621/ articles-116042_archivo_pdf3.pdf(consultado 10.08.2015).

Misses-Liwerant, JuditBokser (2014, Enero-abril), “Los desafíos de las ciencias sociales frente a las múltiples resonancias de lo global”. Revista mexicana ciencias políticas y sociales, Vol. 59. № 220. Recuperado dehttp:/ /www.elsevier.es/es-revista-revista-mexicana-ciencias-politicas-sociales-92articulo-los-desafios-las-ciencias-sociales-90371855 (consultado 10.08.2015).

Ortiz, Renato (20 de Abril de 2011), "Las Ciencias Sociales frente a los desafíos del nuevo siglo”. El Diario. Recuperado de http:// www.eldiario.com.ar/diario/interes-general/2941-las-ciencias-sociales-frentea-los-desafios-del-nuevo-siglo.htm (consultado 10.08.2015).

Pacheco Ladrón de Guevara, Lourdes C. (2009, enero-junio), “Retos de las Ciencias Sociales en las universidades públicas”. Nóesis. Revista de Cien- 
cias Sociales y Humanidades. Vol. 18, º 35, 89-102. México: Instituto de Ciencias Sociales y Administración. Recuperado de http://www.redalyc.org/ articulo.oa?id=85916757008 (consultado 10.08.2015).

Piola, María Eugenia (2000, 08, 01), “Innovación, desarrollo y medio local. Dimensiones sociales y espaciales de la innovación”. Scripta Nova. Revista Electrónica de Geografía y Ciencias Sociales. No 69 (80). España: Universidad de Barcelona. Recuperado de http://www.ub.edu/geocrit/sn-69-80.htm (consultado 10.08.2015).

Plata C, Juan José (2009), “Confianza: el reto para las ciencias sociales”. Revista de derecho $\mathrm{N}^{\circ}$ 31, 259-279. Colombia: Universidad del Norte. Recuperado de http://www.redalyc.org/articulo.oa?id=85112614010 (consultado 10.08.2015).

Ramírez R, Pamela (septiembre 15 de 2010), Las disciplinas de las Ciencias Sociales y sus desafíos. Facultad de Ciencias Sociales de la Universidad de Chile. Recuperado de http://www.facso.uchile.cl/noticias/65534/las-disciplinas-de-las-ciencias-sociales-y-sus-desafios(consultado 10.08.2015).

Retamozo, Martín, Morris, María Belén y Bracco, Olga (2014, julio-diciembre), "Los desafíos de las ciencias sociales y el pensamiento político en América Latina (algunas notas)”. Oficios Terrestres N 31, 60-76. Recuperado de http://perio.unlp.edu.ar/ojs/index.php/oficiosterrestres/ index(consultado 10.08.2015).

Rodríguez Díaz, Erwin (2013, mayo-agosto), “La relación entre el tiempo largo y el tiempo corto. Un intento por revalorar a un pariente pobre de las Ciencias Sociales: la coyuntura”. Estudios Políticos N²9, 149-170. México: Universidad Nacional Autónoma de México. Recuperado de http:// www.sciencedirect.com/science/article/pii/S0185161613726532(consultado 10.08.2015).

Rodríguez Becerra, Manuel, (2012), “Un informe contundente: la gestión minero-ambiental al banquillo”, Revista: Economía Colombiana, Contraloría General de la República. Edición 334, Febrero-Marzo, 2012, páginas 5871.

Serra, Luis (2008), “Retos de las ciencias sociales en Nicaragua”. Encuentro. Vol 40, N 79, 52-62. Recuperado de http://www.uca.edu.ni/encuentro/ images/stories/2012/pdf/79e/79e7a.pdf(consultado 10.08.2015).

Stern Review, The Economics of Climate Change, Executive Summary, http:/ /siteresources.worldbank.org/INTINDONESIA/Resources/2262711170911056314/3428109-1174614780539/SternReviewEng.pdf(consultado 10.08.2015).

The living Planet Report 2014 (2014), WWF, http://ba04e385e36eeed47f9cabbcd57a2a90674a4bcb7fab6c6198d0.r88.cf1.rackcdn.com/ 
Living_Planet_Report_2014.pdf (consultado 10.08.2015).

Tudela Poblete, Patricio (2011), Aportes y desafíos de las ciencias sociales a la organización y la actividad policial. Fundación paz ciudadana, Santiago de Chile. Recuperado de http://www.pazciudadana.cl/wp-content/uploads/ 2011/01/aportes-y-desafios-de-las-ciencias-sociales.pdf(consultado 10.08.2015).

Universidad de los Andes (2013), "Pertinencia y calidad: la Facultad de Ciencias Sociales frente a los retos de Colombia”. Revista Latitudes, Facultad de Ciencias Sociales. Recuperado de http://sextante.uniandes.edu.co/ index.php/ejemplares/sextante-2/latitudes/pertinencia-y-calidad-la-facultadde-ciencias-sociales-frente-a-los-retos-de-colombia(consultado 10.08.2015).

Valencia Gutiérrez, Alberto (2014, enero-junio), “Grandes retos de las ciencias sociales contemporáneas”. Revista colombiana de Sociología. Vol.37 (1), 157-175.Colombia: Universidad del Valle. Recuperado dehttp:// www.revistas.unal.edu.co/index.php/recs/article/view/44619/45931(consultado 10.08.2015).

Vasco U, Carlos E. (10 de Marzo de 2006), “Siete retos de la educación colombiana para el período de 2006 a 2019”. Eduteka, Universidad EAFIT. Recuperado de http://www.eduteka.org/RetosEducativos.php(consultado 10.08.2015).

Walsh, Catherine (2007, abril), “¿Son posibles unas ciencias sociales/culturales otras? Reflexiones en torno a las epistemologías decoloniales”. Nómadas, Universidad Central $\mathrm{N}^{\circ}$ 26, 102-113. Recuperado de http:// www.ucentral.edu.co/images/editorial/nomadas/docs/ nomadas_8_son_posibles_catherine.pdf(consultado 10.08.2015).

Recibido: 10.08.2015

Aceptado: 25.08.2015 